\title{
Percepción docente en los programas de formación para lograr calidad educativa en escuelas secundarias marginadas
}

\author{
José Andrés Castillo Hernández a
}

Resumen - Conocer la percepción docente hacia los programas de formación que pudieran contribuir a en la educación de calidad para favorecer el desarrollo en las escuelas. Los alumnos de esos contextos socioeconómicos presentan condiciones de vulnerabilidad, por lo que los esfuerzos de la sociedad y de los educadores debe estar enfocada en buscar los medios que permitan impartir educación de calidad, que cumpla el precepto de equidad y sobre todo contextualizada a los requerimientos históricos de la educación en sus programas oficiales.

Palabras clave - Percepción, Calidad Educativa, Secundarias Marginadas.

Abstract - Know the teaching perception towards the training programs that could contribute to a quality education that favors the development in the schools. Students from these socio-economic contexts have conditions of vulnerability, so the efforts of society and educators should be focused on finding the means to provide quality education that meets the precept of equity and above all contextualized to the requirements of education in their official programs.

\section{CÓMO CITAR}

HOW TO CITE:

Castillo-Hernández, J. A. (202I). Percepción docente en los programas de formación para lograr calidad educativa en escuelas secundarias marginadas. Interconectando Saberes, (12), 39-48. https://doi.org/I0.25009/is.v0il 2 .2701

Recibido: II de marzo de 202 I Aceptado: 5 de mayo de 2021 Publicado: 20 de julio de 2021

Keywords - Perception, Educational Quality, High School Marginalized.

a Universidad Americana de Europa, México. E-mail: andrescastillo37@hotmail.com 


\section{INTRODUCCIÓN}

El Fondo Internacional de Emergencia de las Naciones Unidas para la Infancia (UNICEF, 2015) plantea que la educación busca la equidad al ofrecer las oportunidades para adquirir conocimientos generales y actitudes positivas ante la vida. En el ámbito escolar se pide que se preparen efectiva y eficientemente los alumnos, es decir que adquieran competencias para la vida, sobre todo a los grupos más vulnerables y marginados, para intentar tener sociedades equitativas, homogéneas y democráticas. La equidad educativa trata y que los alumnos obtengan oportunidades para el desarrollo de sus potencialidades (Secretaria de Educación Pública [SEP], 20I7).

La educación es el vínculo hacia una sociedad con equidad, sin embargo, esto no sucede en la mayoría de las ocasiones, los integrantes de los grupos sociales vulnerables o marginados, no rompen el circulo social al cual pertenecen en su mayoría dando como resultado un rezago en cada uno de los aspectos contextuales que les rodean.

La escuela es importante en la equidad, respecto a la competitividad y la economía, como en su función mediadora entre la equidad y justicia social (Pozner, 2000). Para alcanzar la equidad, por medio de la educación el trabajo conjunto de autoridades educativas, padres de familia, docentes y alumnos, con el apoyo de la sociedad a la que se pertenece, es necesario en una educación transformadora y se alcancen objetivos planteados tanto por las autoridades educativas del país (SEP, 2016, 2017).

Los alumnos que se encuentran en desventaja al estar en una sociedad marginada, se caracterizan por una educación y cultura limitadas, lo que se pretende es romper con este círculo vicioso que la educación sea la transformadora y modifique su estatus social y forma de vida, aunque hay que romper con la costumbre de que no se da importancia a la educación, cultura y ejemplos positivos en estas sociedades, tal vez porque es apremiante resolver los aspectos de supervivencia inmediatos a lo que se pudieran considerar comodidades fuera de su contexto como el conocimiento (Silas, 2008).

El informe de la Organización para la Cooperación y el Desarrollo Económicos (OCDE, 2016) da cuenta de que $11 \%$ de la variación en el rendimiento en ciencias es directamente atribuible al estatus socioeconómico y que los estudiantes de estatus bajos tienen muchas posibilidades de no alcanzar las competencias básicas en ciencias. En ese sentido, Silas (2008), considera que los factores socioeconómico y cultural de las familias, así como los maestros y la calidad de las interacciones que se logran en la escuela tienen un peso importante en el logro académico de los alumnos.

El término calidad educativa es difícil de precisar, en términos generales se trata de desarrollar competencias en el alumnado y en el profesorado en una equidad en la cual los dos actores tienen la misma importancia y solo se distinguen por el rol que desempeñan.

El sistema educativo al actualizarse en los programas mediados por el contexto, sociedad, situación política, programa educativo oficial y las adecuaciones en del sistema con las TIC en su papel de mediadoras en el proceso educativo, en muchos casos hacen que sea necesario analizar si los conocimientos que se tienen son suficientes, tanto en lo disciplinar como en lo pedagógico, así como en la manera de interactuar efectivamente entre colegas. Una educación 
de calidad deberá enseñar además de los conocimientos formales, formación en valores, para tener como resultados ciudadanos íntegros que puedan incluirse en las sociedades del conocimiento y puedan vivir en democracia (SEP, 2016).

Valenzuela (2004) dice que la calidad educativa es equiparable a los mejores programas de educación que se puedan ofrecer a los alumnos, que les permitan incorporarse de manera efectiva a esta sociedad del conocimiento, aunque las sociedades marginadas no tienen en su mayoría las herramientas de estas y su proceso de integración es parcial, lo cual que exige una adecuación de los conocimientos de manera constante, además de que aprendan a trabajar de forma colaborativa y respeto de los derechos dela sociedad y del contexto, y tengan una incorporación adecuada en su micro sociedad.

Logrará la calidad de la educación coadyuvará a revertir la desigualdad social, la cual en la cual está implicada los movimientos sociales y económicos de cada estado (Vázquez, Pérez, Barriga, 20l4). Una sociedad no puede avanzar de forma equitativa sin un sistema educativo que sea incluyente, democrático y que ofrezca igualdad de oportunidades para la sobrevivencia de la sociedad. (Amar, 2000).

Es necesario integrar una formación escolar efectiva, eficiente y de calidad, sobre todo de los grupos marginados debe ser la prioridad del sistema educativo. Los esfuerzos de la sociedad del conocimiento deben estar encaminados a ese fin. Silas (2008) menciona que hay una conjunción de factores personales, familiares, escolares y comunitarios, que permiten a algunos alumnos de estos contextos y seguir con su formación educativa, la cual es solo la punta del iceberg lo cual nos deja con la incertidumbre de lo que pueda conseguirse realmente al implementar programas educativos inclusivos.

Para lograr esta educación, el papel del docente frente a grupo es fundamental. La repuesta del docente no es dependiente tan solo de sus competencias como lo son: vocación, manejo de grupo o aplicación de contenidos curriculares. Primordialmente debe articular en sus clases una planeación que se fundamente en planes y programas de estudio, y la reflexión pedagógica grupal entre docentes para aprender los activos intangibles de los elementos más destacados y la actualización permanente, para homogeneizar el nivel del capital humano a un nivel más elevado (Pozner, 2000).

La cultura del trabajo colaborativo deberá llevarse a la práctica con los alumnos, para que participen de manera activa, como señala Shabel (20l4), aprender a trabajar con los alumnos para que puedan integrarse de manera activa a la sociedad, desde una perspectiva participativa, desde una metodología etnológica, y como el objetivo de construcción bilateral del conocimiento.

Esta educación podrá ser posible si se innova de manera permanente y promueve el desarrollo integral del alumno, que le permita estar preparado para aprender por su cuenta, esto es como relatan Suárez, Almerich, Gargallo y Aliaga (2013), en sintonía con las exigencias de los ciudadanos desarrollarse en sociedad del conocimiento la cual es una utopía que se de en todos los habitantes del planeta, aun así es menester del docente romper los paradigmas y transformar la sociedad uno a uno y enriquecer la vida de los participantes de esta. 
Para plantear esta situación, hay que especificar que la educación es el único medio por el cual los individuos pueden transformarse y transformar su comunidad (UNICEF, 20I5), es menester que se dé una educación con calidad y posibilite a los alumnos primeramente entender la situación histórica por la que están atravesando, esto es la realidad que están viviendo y en seguida una preparación eficiente tanto en conocimientos disciplinares, actitudinales y de valores. Solamente una educación de calidad traerá consigo una mejor sociedad en la que sus ciudadanos puedan vivir en armonía entre ellos como con la naturaleza.

Los cambios acelerados que ha traído la globalización del conocimiento están presentes en todos los ámbitos y se espera que alcancen hasta los últimos rincones del planeta, creándose entornos sumamente competitivos, inequitativos e inestables, aunque es la práctica no puede que sea del todo cierto. Las sociedades más desarrolladas tienen acceso a una mejor educación y sus ciudadanos viven mejor, en contraparte los grupos más vulnerables que viven en condiciones de pobreza no pueden acceder a una educación de calidad, lo que genera un círculo vicioso de pobreza y falta de oportunidades educativas (UNICEF, 20I5).

Sirven de antecedente a este planteamiento los resultados de la OCDE (2016), ubican a México en los últimos lugares. En ciencias con $46 \mathrm{I}$ contra el promedio de 493 , en lectura de 423 contra 493, de matemáticas de 408 contra 490 , como se ve todos estos resultados por debajo del promedio. En estas tres áreas menos del I \% alcanzan resultados de excelencia de 5 y 6 , en ciencias no se observan mejoras significativas desde el 2006. Lectura y matemáticas tienen una mejora, que no le alcanza al país para mejorar su ubicación entre los países miembros (OCDE, 20I6).
Y dentro del país, el porcentaje del logro educativo es insuficiente en las materias de español y matemáticas de los alumnos de sexto grado de primaria en Oaxaca evaluados por los exámenes Excale en el año 2005 fue de 23.0, sólo por arriba de los estados de Guerrero (28.0) y Chiapas (3।.2), no hay resultados para el 2009 para estos estados porque no se aplicaron esos exámenes (INEE, 2012). Para los alumnos indígenas de secundaria sólo el 38 por ciento tiene ese nivel terminado a los 16 años según CONEVAL (2010, citado por CONEVAL, 20I I, p. I5).

Definiendo esto los alumnos de las comunidades marginadas generalmente se quedan sin acceder a la educación de calidad (Schmelkes, 2005), a pesar de los programas que ha implementado el gobierno se siguen dando resultados bajos en eficiencia terminal y en el porcentaje de aprovechamiento, ante este panorama es necesario buscar los mecanismos que posibiliten un mejor nivel educativo en estas sociedades, para ofertar una mejor preparación y con ello garantizar la inclusión de estos alumnos en la nueva sociedad del conocimiento.

Los factores que permiten o frenan la calidad y pertinencia en el proceso de enseñanza aprendizaje son múltiples, a saber, intervienen los: alumnos, padres de familia, docentes, planes y programas, la infraestructura educativa y el contexto. La forma en que entre ellos se interrelacionan es compleja. Resulta relevante investigar lo concerniente a la percepción docente a la relación entre los programas oficiales de capacitación en el logro de la educación de calidad, medida comúnmente entre los directivos a través de los resultados en promedios de aprovechamiento, porcentajes de aprobación y permanencia en la escuela. 


\section{MÉTODO DE INVESTIGACIÓN}

Para esta investigación se eligió la metodología mixta, la cual permite utilizar los dos paradigmas fundamentales, el positivista (método cuantitativo) y el naturalista (método cualitativo), de esta manera es posible estudiar aspectos apropiados para cada enfoque. Hernández-Sampieri, Fernández-Collado \& BaptistaLucio (20l4) y Pérez, Galán, Quintanal (20I2) argumentan que los dos tipos de métodos brindan una comprensión que no podría dar un método por separado y que, los métodos no se encuentran libres de la percepción del docente y esto implica ventajas y limitaciones, es un acercamiento a la verdad circunstancial del medio en donde se aplica y esto lleva al investigador a proponer una visión única del tema.

\section{PREGUNTA DE INVESTIGACIÓN}

¿Cuál es la percepción de los docentes de secundaria con respecto a la relación entre los programas oficiales de capacitación y una educación de calidad?

\section{OBJETIVO DE LA INVESTIGACIÓN}

Identificar datos relevantes a partir de los instrumentos propuestos, que permitan responder si los docentes consideran importantes los programas oficiales de capacitación y si a partir de ellos se puede impartir una educación de calidad.

\section{POBLACIÓN Y MUESTRA}

Para la parte cuantitativa, se eligieron los alumnos participantes por medio de un muestreo probabilístico estratificado, que permite aprovechar las características de la población (Elorza, 2008 y Valenzuela y Flores, 20I I), los estratos se hicieron por grados, por población origen de los alumnos (Santa María Nutio, El Terrero y Nuevo Progreso) e integrantes de la sociedad de alumnos tres alumnos por grado, más tres integrantes de la sociedad de alumnos (uno por grado y de cada población), en total 12 alumnos, siendo 4 por grado y por lugar de origen. Dentro de cada estrato se hizo una tómbola para seleccionar al alumno participante (Hernández-Sampieri, Fernández-Collado \& Baptista-Lucio, 2014)

\section{INSTRUMENTOS}

Los instrumentos aplicados son de autores reconocidos en el tema, para asegurar la validez, el apéndice $\mathrm{Al}$ es un cuestionario-escala de Traver y García (2007) que mide la actitud del docente frente respecto a la innovación educativa. Un cuestionario de una investigación de Fernández y Sánchez (2014) que mide las competencias profesionales del docente de secundaria.

Para conocer los puntos de vista de los alumnos se utilizaron tres cuestionarios. Uno para evaluar las características de los profesores de Fernández y Sánchez (20I4), otro para evaluar el curso en general), tomando como base el cuestionario de Valenzuela (2004) y el último para medir lo que piensan con respecto a las matemáticas de Vallejo y Escudero (I999). La materia de matemáticas tiene un alto grado de reprobación por ello se incluyó el último instrumento para saber que piensan los alumnos de esta materia.

La forma de evaluar los instrumentos fue tomada del trabajo de Fernández y Sánchez (20l4). Todos los cuestionarios se evaluaron con una escala Likert y sus resultados fueron contrastados con los de Fernández y Sánchez (20|4). 


\section{PROCEDIMIENTO}

Se llevó a cabo el proceso de recolección de información fue la siguiente:

- Fase I. Se solicitó la autorización por escrito a la supervisión escolar para informarle acerca de este trabajo, los instrumentos que se aplicarían, y el beneficio que le traería a la Escuela.

- Fase II. Se estableció la muestra estratificada de alumnos por población de origen y por grado, se diseñaron los horarios para aplicar los cuestionarios y las observaciones etnográficas.

- Fase III. Se les informó a los participantes sobre los cuestionarios y la observación etnográfica y cuál sería el objetivo. A los docentes se les aplicó el cuestionario en su salón a los alumnos se les aplicaron los cuestionarios en diferentes momentos.

- Fase IV. Se calificaron los cuestionarios. Los resultados del instrumento del Apéndice B2, indicaron los grupos y materias en donde se realizaron las observaciones etnográficas.

- Fase V. Se realizaron las observaciones etnográficas con el propósito de recolectar la información para analizar y comprender y así responder a la pregunta de investigación y producir conocimiento (Hernández-Sampieri, Fernández-Collado \& Baptista-Lucio, 2014).

- Fase VI. Se revisaron las planeaciones didácticas docentes

- Fase VII. Se trianguló la información para encontrar los hallazgos de la investigación (Hernández-Sampieri, Fernández-Collado \& Baptista-Lucio, 20|4).
Para analizar cuantitativamente todos los cuestionarios se utilizó una escala ordinal tipo Likert, con 5 posibles respuestas que comprenden los niveles: muy de acuerdo (I), de acuerdo (2), no tiene una opinión definida (3), no está de acuerdo (4), está muy en desacuerdo (5), asignándoles los valores de 3, 2, I y 0 a (I), (2), (4) y (5) respectivamente, y al valor (3) que no tiene una opción definida no se le asignó valor numérico (Hernández-Sampieri, Fernández-Collado \& Baptista-Lucio, 2014), para obtener indicadores numéricos estadísticos, para encontrar el valor promedio (Med), la desviación típica ( $\mathrm{Sx}$ ) y el coeficiente de variación $(\mathrm{CV})$. Para encontrar la desviación se tuvo que calcular la varianza. Estos datos se confrontaron con los resultados de las observaciones y con los resultados bimestrales.

En los métodos mixtos la triangulación de resultados se realiza para combinar las fortalezas de ambas metodologías y dar respuestas complementarias acerca de un mismo problema de investigación para dar validez a los resultados de esta (Pereira, 20l I). Esta se realizó a través de un análisis inductivo, que va de lo particular a lo general, transcribiendo notas de la observación para generar temas comunes. Agrupados en categorías con información similar como: compromiso docente y de los alumnos, didácticas, trabajo individual y de equipo, innovaciones didácticas, motivación de alumnos, que permitieron comprender los resultados $y$ dar respuestas a las preguntas de investigación (Hernández- Sampieri, Fernández-Collado \& BaptistaLucio, 2014).

Finalmente se aplicó un modelo convergente para analizar de manera separada los datos cuantitativos y cualitativos obtenidos para poder encontrar las conclusiones y para darle validez se triangularon los 
resultados obtenidos estadísticamente con los obtenidos a través de la observación etnográfica y el análisis de documentos oficiales.

\section{RESULTADOS}

Lo obtenido a través de los instrumentos planteados en el capítulo referente al método para responder a la pregunta ¿Cuál es la percepción de los docentes de secundaria con respecto a la relación entre los programas oficiales de formación y una educación de calidad?

\section{Respuestas a la pregunta de investigación}

¿Cuál es la percepción de los docentes de secundaria con respecto a la relación entre los programas oficiales de capacitación y una educación de calidad?

Los docentes percibieron que la innovación, así como conocer y aplicar las competencias era importante en la calidad educativa, no obstante, las evidencias analizadas mostraron que un predominio de trabajo conductista individualizado y sin innovaciones (excepto historia), lo cual denotó incongruencia en la práctica educativa.

\section{Preguntas subordinadas}

I. ¿Es congruente lo que piensan los docentes que debe ser una buena práctica educativa con su desenvolvimiento en el aula?

En general los docentes están convencidos de esto, aunque sus prácticas y el discurso a veces dista.

2. ¿De qué forma consideran los profesores de secundaria que los programas de capacitación pueden promover una educación de calidad?
Les dan una importancia alta a las innovaciones pedagógicas, específicamente en el uso de las TIC, se siguen dando clases siguiendo el modelo conductista e individualizado.

3. ¿Influye en el logro de una educación de calidad que los docentes conozcan y consideren importantes las competencias?

Existe influencia, es necesario que tengan claridad conceptual en la aplicación de la educación de calidad y competencias

4. ¿De qué forma consideran los profesores de secundaria que se puede integrar el desarrollo de las competencias grupales y el uso de las TIC a través de un programa de capacitación docente que busque promover una educación de calidad?

Se puede integrar el desarrollo de las competencias grupales y el uso de las TIC a través de un programa de capacitación docente para promover una educación de calidad.

5. ¿Cómo consideran los profesores de secundaria la relación entre la percepción que tienen sus alumnos de su labor instruccional y el logro de los aprendizajes de calidad?

Conocer la valoración que tienen los alumnos de los docentes y en grado esto influye en el logro de aprendizajes de calidad.

\section{Responder los objetivos específicos}

a) Saber lo que piensan los docentes que debe ser una buena práctica educativa y si la llevan a cabo en el aula.

Conocer como consideran los profesores de secundaria que los programas de capacitación docente pueden promover una educación de calidad. 
b) Los docentes percibieron que la innovación, así como conocer y aplicar las competencias es primordial en la calidad educativa.

Encontrar la relación entre conocer y considerar importantes las competencias y el logro de una educación de calidad.

c) Esta se mostró en los docentes mejor valorados con un compromiso con su quehacer docente y consistencia en su desempeño, utilizando innovaciones en clases relacionadas con el uso de la tecnología.

Conocer como consideran los profesores de secundaria que se puede integrar el desarrollo de las competencias grupales y el uso de las TIC a través de un programa de capacitación docente para promover una educación de calidad.

d) Al establecer mejores prácticas educativas se observó que las actitudes de los alumnos en sus clases eran positivas, corroborando que las competencias y capacidades docentes son fundamentales para que se alcance la calidad educativa, ya que los alumnos son muy receptivos y le dan una buena valoración a la forma de enseñanza de estos maestros.

Conocer si los docentes entienden y aplican las competencias del saber, saber hacer, saber estar y saber ser y si esto repercute en el logro de la educación de calidad.

e) Estos necesitan dominar los recursos tecnológicos, saber que recursos necesitan, en cuál forma y cuando utilizarse en la práctica diaria, para ello debe darse una formación que permita adquirir estas competencias. Por lo que si se quiere alcanzar una educación de calidad se deberán implementar programas de formación que apoye a los docentes que tengan áreas de oportunidad en este aspecto.

f) Conocer la valoración que tienen los alumnos de los docentes y en grado esto influye en el logro de aprendizajes de calidad.

\section{CONCLUSIONES}

No todos los docentes desempeñan una buena práctica pedagógica.

La capacitación será muy importante para el logro de mejores prácticas educativas, que permitan alcanzar la calidad educativa, será necesario buscar los mecanismos de transmisión de conocimientos de los docentes mejor valorados para que se dé la transferencia de activos intangibles, se pueda elevar el valor del capital humano de la institución y con ello se logren aprendizajes de calidad.

Será de fundamental importancia diseñar acciones encaminadas a que estos docentes adquieran competencias.

Las áreas de oportunidad que se deberán cubrir son:

I) Reconocimiento de la teoría pedagógica desde donde se desarrolla la práctica educativa actual.

2) Apropiarse de los conocimientos para trabajar desde el constructivismo y/o la pedagogía crítica.

3) Desarrollar las competencias del saber, para planear e introducir prácticas educativas innovadoras, como trabajo colaborativo y uso de las TIC.

4) Recopilar y transmitir los conocimientos de los elementos más capacitados. 
5) Concientizar que una práctica educativa sin compromisos les quita oportunidades a los alumnos.

\section{REFERENCIAS}

Amar, J. (2000). La función social de la educación. Investigación y desarrollo, (I I), 74-85. http://www.redalyc.org/pdf/268/2680 I I 04.pdf

Consejo Nacional de Evaluación de la Política de Desarrollo Social (20I I). Informe de evaluación de la política de desarrollo social en México en materia de rezago educativo.

http://www.coneval.org.mx/Informes/Evaluacion/ Documentos\%20tem\%C3\%AI ticos\%20IEPDS20I I/IEPDS_Educacion_201 I.pdf

Elorza, H. (2008) Estadística para las ciencias sociales, del comportamiento y de la salud ( $3^{\mathrm{a}}$ ed.) Distrito Federal, México, Cengage Learning.

Fernández, J. M. (2009). Las tecnologías de la información y la comunicación desde la perspectiva de la psicología de la educación. Educación y tecnología: DOI: https://doi.org/I0.13|40/2.1.1692.3682

Fernández, R., Sánchez, L., (20l4) Competencias docentes en secundaria. Análisis de perfiles de profesorado. Relieve, 20(I), I-20, http://www.redalyc.org/pdf/916/91632161002.pdf

García, I. V., (20I0). Liderazgo distribuido, una visión innovadora de la dirección escolar: una perspectiva teórica. Omnia, 16(3), 19-36, http://www.redalyc.org/articulo.oa?id=737/6205 003

Hernández Sampieri, R., Fernández Collado, C. \& Baptista Lucio, M. P. (20I I). Metodología de la investigación (6a. ed.). McGraw-Hill.

INEE (20I2). Panorama educativo de México. Indicadores del sistema educativo nacional, Educación básica y media superior. http://publicaciones.inee.edu.mx/buscadorPub/PI/ B/I09/PIBI09.pdf

Organización para la Cooperación y el Desarrollo Económicos. (2016). Programa para la evaluación internacional de alumnos (PISA) 2015. Resultados. https://www.oecd.org/pisa/PISA20I5-Mexico-ESP.pdf
Pereira, Z. (20I I). Los diseños de método mixto en la investigación en educación. Una experiencia concreta. Revista electrónica Educare. I5(I), I529 ,

https://dialnet.unirioja.es/descarga/articulo/36835 44.pdf

Pérez, R., Galán, A., \& Quintanal, J. (2012). Métodos y Diseños de Investigación en Educación. Madrid, España: Universidad Nacional de Educación a Distancia.

Pozner, P. (2000) Competencias para la profesionalización de la gestión educativa. Diez módulos destinados a los responsables de los procesos de transformación educativa, módulo I. Desafios de la educación, Instituto Internacional de Planeamiento de la Educación UNESCO, Buenos Aires. www.buenosaires.iipe.unesco.org/publicaciones/c ompetencias-para-la-profesionalizaci-n-de-lagesti-n-educativa

Sánchez, A., Melián, A. \& Hormiga, E. (2007) El concepto de capital intelectual y sus dimensiones. Red de Revistas científicas de América Latina, I3(2), 97-I I I.

http://www.redalyc.org/pdf/274I/274I 20280005. pdf

SEP (2016). El Modelo Educativo 2016. El planteamiento pedagógico de la reforma educativa. https://sep.gob.mx/work/models/sep I/Resource/ 8007/I/images/mod elo_educativo_2016.pdf

SEP (20I7). Modelo educativo para la educación obligatoria. Educar para la libertad y la creatividad.

https://www.gob.mx/cms/uploads/attachment/file/ 198738/Modelo

Educativo_para_la_Educacio_n_Obligatoria.pdf

Shabel, P. (20I4). Los niños y niñas como

constructores de conocimiento: un caso de investigación participativa. Revista latinoamericana de Ciencias Sociales, Niñez y Juventud, I2(I), I59I70,

http://www.scielo.org.co/pdf/rlcs/v|2nI/v|2nla0 9.pdf

Silas, J. C. (2008). ¿Porque Miriam si va a la Escuela? Resiliencia en la educación básica mexicana. Revista Mexicana de Investigación Educativa, I3(39), I255-1279, http://www.scielo.org.mx/pdf/rmie/v|3n39/v/3n3 9a10.pdf 
Suárez, J. M., Almerich, G., Gargallo, B. \& Aliaga F.

(20I3). Las competencias del profesorado en tic: Estructura básica. Educación XXI. I6(I), 39-62. http://revistas.uned.es/index.php/educacionXXI/a rticle/view/7/6

Traver, J. A., García, R. (2007) Construcción de un cuestionario-escala sobre actitud del profesorado frente a la innovación educativa mediante técnicas de trabajo cooperativo (CAPIC). Revista electrónica de Investigación Educativa, 9(I), I-I4.

http://redie.uabc.mx/redie/article/viewFile/I55/26 7

UNICEF (2015). El argumento en favor de la inversión en la educación y la equidad. Resumen ejecutivo. https://www.unicef.org/ecuador/La_educacion_y la_equidad_2015(I).pdf

Valenzuela, J. R. (2004). Evaluación de Instituciones Educativas. (reimp. 20I2). Trillas.

Valenzuela, J. R. y Flores, M. (20I I). Fundamentos de investigación educativa (eBook). Editorial Digital Tecnológico de Monterrey.

Vallejo, G. \& Escudero, J. R. (1999). Cuestionario para evaluar las actitudes de los estudiantes de E.S.O. hacia las matemáticas. Revista Aula Abierta, 74(I), I-I6.

https://dialnet.unirioja.es/servlet/articulo?codigo= $\underline{45466}$

Vázquez, V., Pérez, T., Díaz, F. (20|4). El caso de Juan, el niño Triqui. Una experiencia de formación docente en educación intercultural. Revista Mexicana de Investigación Educativa, 19(60), 129I54, http://www.scielo.org.mx/pdf/rmie/vl9n60/v19 n60a7.pdf 